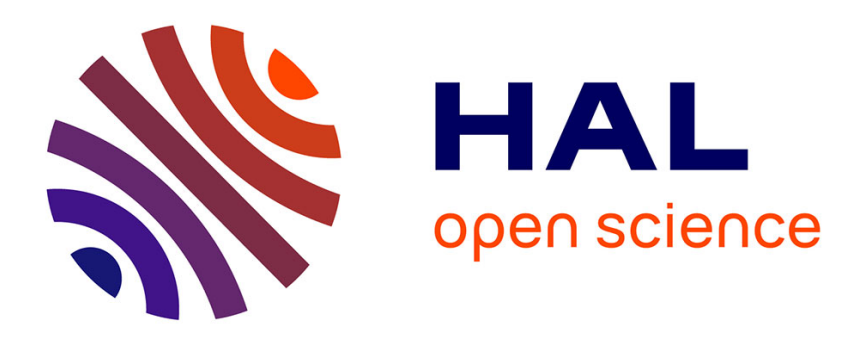

\title{
Réduction des émissions des CO2 chinoises et gaz de schiste
}

\author{
Marie-Hélène Hubert
}

\section{To cite this version:}

Marie-Hélène Hubert. Réduction des émissions des $\mathrm{CO} 2$ chinoises et gaz de schiste. Revue Française d'Economie, 2017, XXXI (3), pp.51. 10.3917/rfe.163.0051 . halshs-02315562

\section{HAL Id: halshs-02315562 \\ https://shs.hal.science/halshs-02315562}

Submitted on 21 Oct 2019

HAL is a multi-disciplinary open access archive for the deposit and dissemination of scientific research documents, whether they are published or not. The documents may come from teaching and research institutions in France or abroad, or from public or private research centers.
L'archive ouverte pluridisciplinaire $\mathbf{H A L}$, est destinée au dépôt et à la diffusion de documents scientifiques de niveau recherche, publiés ou non, émanant des établissements d'enseignement et de recherche français ou étrangers, des laboratoires publics ou privés. 


\title{
Réduction des émissions des $\mathrm{CO}_{2}$ chinoises et gaz de schiste
}

\author{
Marie-Hélène Hubert
}

Novembre 2016

\section{Résumé :}

Afin de diminuer ses émissions de $\mathrm{CO}_{2}$, le gouvernement chinois vise à accroître la part du gaz, notamment, en extrayant les réserves de gaz de schiste chinoises. Le gaz de schiste peut fournir $10 \%$ de la consommation d'énergie d'ici 2030 si son coût d'extraction diminue d'un tiers. Toutefois, les émissions de $\mathrm{CO}_{2}$ chinoises augmentent de $5 \%$. Une politique imposant la stagnation de la consommation de charbon favorise l'extraction du gaz de schiste à grande échelle et diminue les émissions de $\mathrm{CO}_{2}$ de $41 \%$. En revanche, une taxe sur les émissions de $\mathrm{CO}_{2}$ ralentit le développement du gaz de schiste au profit des énergies renouvelables.

Mots-Clés: Gaz de schiste, Changement climatique, Marché de l'énergie

JEL Codes: Q33, Q38, Q42

\section{Abstract:}

To reduce $\mathrm{CO}_{2}$ emissions, Chinese government sets a target on the share of gas in its energy consumption. Shale gas can supply around $10 \%$ of energy consumption if its cost declines by one third, but it drives up domestic $\mathrm{CO}_{2}$ emissions by 5\%. A policy aiming at stabilizing coal consumption leads to large development of shale gas as well as a decrease in $\mathrm{CO}_{2}$ emissions of $41 \%$. However, a tax on $\mathrm{C} 02$ emissions slows down shale gas development in favor of renewable energy.

Keywords: Shale gas, Climate change, Energy markets

\section{Introduction}

\footnotetext{
* CREM, Faculté de Sciences Économiques, 7 Place Hoche 35065 Rennes, FRANCE. mariehelene.hubert@univ-rennes1.fr. Je remercie Gilles Lafforgue et un rapporteur anonyme pour la qualité et la pertinence de leurs suggestions qui ont contribué à améliorer le papier. Je remercie le Conseil Français de l'Énergie pour le financement du projet : L'impact des stratégies énergétiques des pays émergents sur les émissions mondiales de $\mathrm{CO}_{2}$.
} 
Le gaz de schiste est une ressource non conventionnelle au même titre que le sable bitumineux et le pétrole de schiste. Il est piégé dans des roches marneuses ou argileuses, enfouies généralement entre 1500 et 3000 mètres de profondeur. ${ }^{2}$ Son extraction nécessite l'usage d'une technologie particulière appelée fracturation hydraulique. ${ }^{3}$ Le gaz de schiste extrait est raffiné sur place avant d'être transporté pour stockage ou utilisation directe (via des pipelines) en vue de produire in fine de l'électricité ou d'autres types d'énergie (Jackson, et al., 2014).

\section{Figure 1 : Évolution de la production de gaz conventionnel et de gaz de schiste aux États-Unis, 2002-2015}

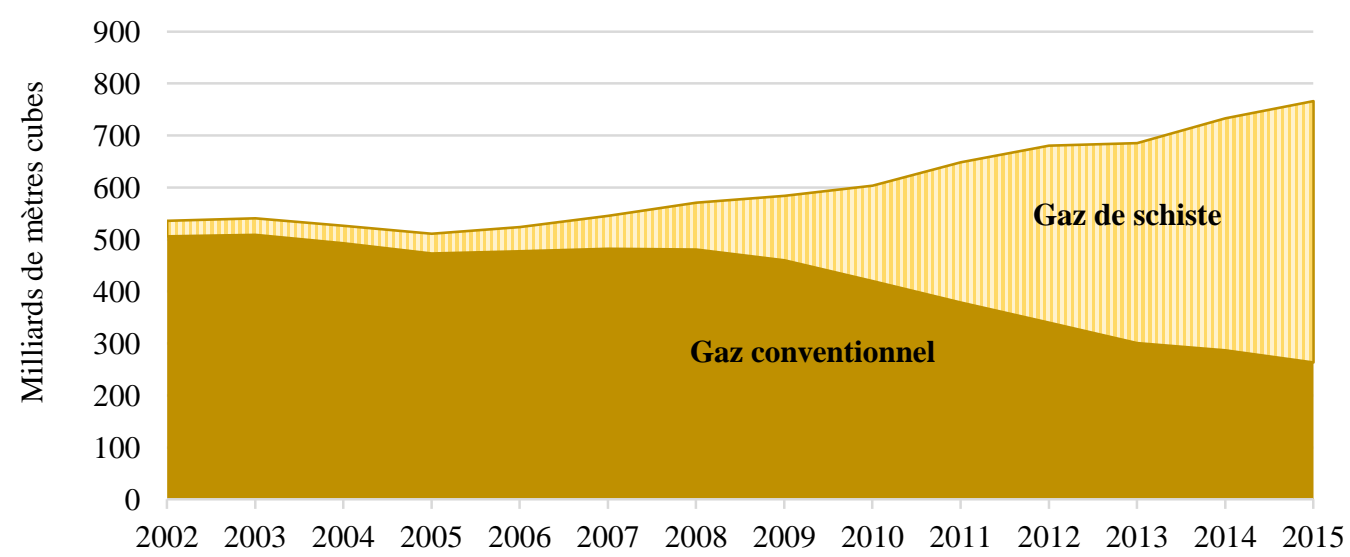

Source: (EIA 2015b)

L'extraction à échelle commerciale du gaz de schiste a débuté aux États-Unis. La production américaine de gaz de schiste, quasi-inexistante en 2006, a atteint 380 milliards de mètres cubes en 2015 soit un peu plus $50 \%$ de la production nationale de gaz (voir Figure 1). La révolution du gaz de schiste a eu des répercussions économiques importantes aux ÉtatsUnis : ré-industrialisation de certaines régions, diminution des importations de ressources naturelles, le prix du gaz est 40\% à 50\% moins cher qu'en Europe. En 2015, seulement quatre pays produisent du gaz de schiste à des niveaux commerciaux : les États-Unis, le Canada, la Chine et l'Argentine (EIA [2015a]). D'après les estimations de l'agence américaine d'information sur l'énergie (EIA), les ressources techniquement récupérables mondiales de gaz de schiste représentent $32 \%$ des ressources techniquement récupérables mondiales de gaz naturel (EIA [2015b]). Près du tiers des ressources de gaz de schiste sont concentrées dans trois pays (la Chine, les États-Unis et l'Argentine), dont $20 \%$ par la Chine seule (voir Figure 2). Toutefois, de fortes incertitudes subsistent sur le montant exact des ressources de gaz de schiste en l'absence d'exploitation à grande échelle.

Figure 2 Répartition des ressources techniquement récupérables de gaz de schiste 


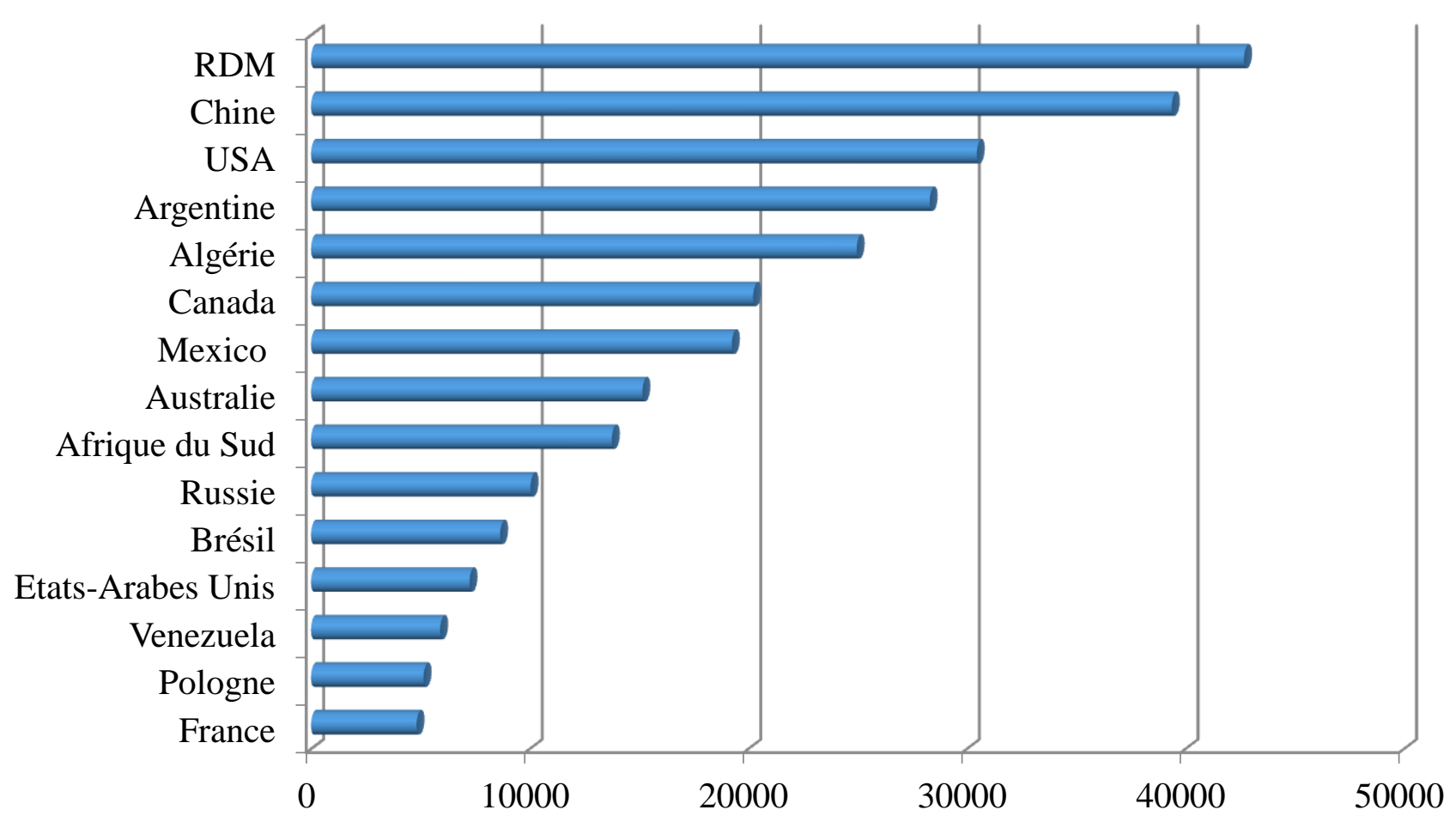

Milliards de mètres cubes

Source : EIA [2013b]. Note : Sont reportées les ressources de gaz de schiste techniquement récupérables recensées par l'Agence Américaine d'Information en 2013. Les ressources mondiales de gaz de schiste s'élèvent à 276040 milliards de mètres cubes, quant à celles de gaz naturel, elles avoisinent 808070 milliards de mètres cubes.

La Chine, deuxième économie mondiale, doit relever le défi de satisfaire les besoins énergétiques de près d'1,4 milliards d'individus tout en réduisant la pollution atmosphérique et en assurant son indépendance énergétique. Elle doit diversifier son portefeuille énergétique qui, en 2013, comportait 60\% de charbon contre seulement $5 \%$ de gaz naturel et, malgré de récents investissements, une part insignifiante de nucléaire. Une des options retenues est le développement du gaz naturel et notamment celui du gaz de schiste. Howarth, Ingraffea et Engelder [2011] et Jackson, et al. [2014] ont prouvé que remplacer le charbon par le gaz pour la production d'une unité d'énergie réduit d'un tiers les émissions de $\mathrm{CO}_{2}{ }^{4}$ En comptabilisant les possibles fuites de méthane lors de l'extraction des ressources fossiles, le gaz émet deux fois plus d'émissions que le charbon pour produire une unité d'énergie (Allen, et al. [2013]).

L'objectif de cette étude est d'analyser l'impact des politiques chinoises visant à diversifier le portefeuille énergétique sur les marchés énergétiques chinois. Notamment, le développement du gaz de schiste en Chine suscite plusieurs interrogations. D'une part, au niveau national, dans quelle mesure le gaz de schiste peut-il remplacer le charbon ? En outre, son développement est-il susceptible de retarder celui des énergies renouvelables et d'induire, selon la terminologie de Fischer et Salant [2012], une fuite de $\mathrm{CO}_{2}$ inter-temporelle ? ${ }^{5}$ D'autre part, la Chine consommant environ la moitié du charbon mondial, toute politique visant à réduire sa consommation devrait diminuer son prix mondial incitant les autres régions du monde à accroitre leur consommation de charbon. Ainsi, une fuite spatiale de $\mathrm{CO}_{2}$ pourrait être observée (ou spatial release effect, Fischer et Salant [2012]). 
Pour répondre à ces questions, un modèle d'équilibre partiel du secteur énergétique mondial est développé sur la base de celui construit par Chakravorty, Fischer, \& Hubert, [2016]. Trois régions, l'Amérique du Nord, la Chine et le Reste du Monde extraient et consomment des ressources fossiles (charbon, pétrole, gaz naturel et gaz de schiste), des ressources renouvelables (hydroélectricité, biomasse et éolien/solaire) et de l'énergie nucléaire afin de satisfaire la demande finale d'énergie de trois secteurs : l'industrie, le secteur commercial/résidentiel et le secteur des transports. Les ressources fossiles sont échangées entre les différentes régions. Les résultats de cette étude montrent que le développement du gaz de schiste en Chine peut être effectif si le gouvernement subventionne fortement la production de cette énergie ou/et si le gouvernement taxe le charbon. Le rôle du gaz de schiste pour satisfaire les objectifs de réduction des émissions de $\mathrm{CO}_{2}$ devrait être minime, les nouvelles énergies vertes comme l'éolien et le solaire devraient jouer un rôle prépondérant. Ces différentes politiques devraient participer à l'accroissement des émissions de $\mathrm{CO}_{2}$ en Amérique du Nord et dans le Reste du Monde (RDM).

Plusieurs études se sont intéressées au rôle du gaz de schiste sur les marchés énergétiques nationaux et mondiaux. Cullen et Mansur [2016] examinent l'effet d'une taxe sur le $\mathrm{CO}_{2}$ dans le secteur électrique américain en examinant le rôle potentiel du gaz de schiste comme substitut au charbon. Ils montrent qu'une taxe de 20 (respectivement de 70) dollars américain par tonne de $\mathrm{CO}_{2}$ devrait réduire les émissions de $\mathrm{C}_{2}$ de $5 \%$ (respectivement de $10 \%$ ). Les effets de la taxe sur le $\mathrm{CO}_{2}$ sont renforcés si le prix du gaz est faible. Henriet et Schubert [2015] développent un modèle d'usage des ressources de type Hotelling afin d'analyser l'arbitrage entre le développement du gaz de schiste et celui des énergies renouvelables en remplacement du charbon si les pollutions locale et globale sont considérées. Selon cette étude, une politique climatique contraignante favoriserait le développement du gaz de schiste et retarderait la transition vers les énergies vertes. Afin d'examiner les effets des fuites du $\mathrm{CO}_{2}$ d'une politique climatique nationale, Daubanes, Henriet et Schubert [2016] construisent un modèle d'équilibre partiel du marché de l'énergie en considérant deux régions qui produisent et consomment deux ressources, le charbon et le gaz de schiste. Toute politique climatique nationale conduit à accroître la consommation domestique de gaz de schiste au détriment de celle du charbon. Les exportations de charbon augmentant, les émissions mondiales de $\mathrm{C}_{2}$ s'accroissent. D'autres études ont tenté de mettre en perspective les facteurs qui ont favorisé la révolution du gaz de schiste aux États-Unis tout en discutant de ses conséquences sur l'économie américaine (Wang et Krupnick [2013]). Enfin, Yuan, et al. [2015] ont analysé les différentes options pour le développement du gaz de schiste en Chine. Notre étude contribue à la littérature naissante sur le développement du gaz de schiste à plusieurs niveaux. D'une part, elle met la lumière sur le rôle potentiel du gaz dans le futur paysage énergétique chinois. D'autre part, elle analyse l'impact de cette politique sur les marchés énergétiques mondiaux.

Le papier est organisé comme suit. La deuxième section détaille la politique énergétique et climatique chinoise. La troisième section présente dans ses grandes lignes le modèle d'équilibre partiel des marchés énergétiques mondiaux construit pour cette étude ainsi que les scénarii de politiques climatiques et énergétiques étudiés. L'impact de ces politiques sur les marchés énergétiques chinois et mondiaux est analysé ainsi que leur bilan $\mathrm{CO}_{2}$ dans la quatrième section. Enfin, la cinquième section conclut et présente les perspectives de recherche. 


\section{La politique énergétique et climatique de la Chine}

Au cours des trois dernières décennies, la croissance chinoise a été spectaculaire : le PIB par habitant a été multiplié par 13 en dollars constant et en parité des pouvoirs d'achat pour atteindre 13400 dollars en 2015. La demande d'énergie par tête a progressé de 190\% de 1990 à 2013 passant de 766 kilogrammes d'équivalent pétrole à 2226 (World Bank [2016]). Les émissions totales de $\mathrm{CO}_{2}$ ont progressé en moyenne de 6\% par an entre 1990 et 2013. En 2008, la Chine est devenue le premier émetteur mondial de $\mathrm{CO}_{2}$, suivie par les États-Unis et l'Union Européenne. Les émissions par tête de $\mathrm{CO}_{2}$ ont également connu une progression fulgurante. En 1990, un citoyen chinois émettait environ 2 tonnes de $\mathrm{C}_{2}$ par an, en 2013, sa consommation a atteint 6,6 tonnes. Malgré cette forte progression, la différence d'émissions par tête entre les deux plus gros pollueurs, i.e., la Chine et les Etats-Unis, est notable. Un citoyen chinois émet environ 10 tonnes de $\mathrm{CO}_{2}$ par an de moins. La croissance chinoise est moins intensive en $\mathrm{CO}_{2}$. En effet, pour produire un dollar de PIB la Chine émettait environ 1,35 kilogrammes d'émissions de $\mathrm{CO}_{2}$ en 1990 contre 0,63 kilogramme en $2013 .{ }^{6}$

La Chine avait ratifié le Protocole de Kyoto. Étant donné qu'elle faisait partie des pays de l'Annexe I, aucune cible de diminution des émissions de $\mathrm{CO}_{2}$ n'avait été définie (Auffhammer et Gong [2015]). La Chine s'est engagée en Novembre 2014 avec la signature d'un accord bilatéral avec les États-Unis à stabiliser ses émissions de carbone d'ici 2030. En Juin 2015, elle a annoncé l'instauration d'une cible de réduction de l'intensité en $\mathrm{C}_{2}$ de $60 \%$ à $65 \%$ par rapport à son niveau de 2005. ${ }^{7}$ Enfin, avec la ratification des accords de Paris en septembre 2016, la Chine s'est engagée à porter la part des énergies non carbonées dans sa consommation primaire d'énergie à $20 \%$. Afin de respecter l'ensemble de ces objectifs tout en continuant à satisfaire ses besoins croissants en énergie, elle doit modifier sensiblement son portefeuille énergétique. Environ $60 \%$ de l'énergie provient du charbon (Voir

Figure 3). La part des énergies renouvelables et celle du nucléaire s'élèvent respectivement à $17 \%$ et à $1 \%$, celle du gaz n'excède pas $5 \%$ en 2013 . Afin de respecter ses objectifs de diminution des émissions de gaz à effet serre, la Chine poursuit des politiques en faveur des énergies renouvelables mais aussi en faveur du gaz et du nucléaire. Plus récemment, elle a établi une feuille de route pour le développement de la séquestration géologique du $\mathrm{CO}_{2}$. 


\section{Figure 3 : Portefeuille Énergétique de la Chine, Année 2013}

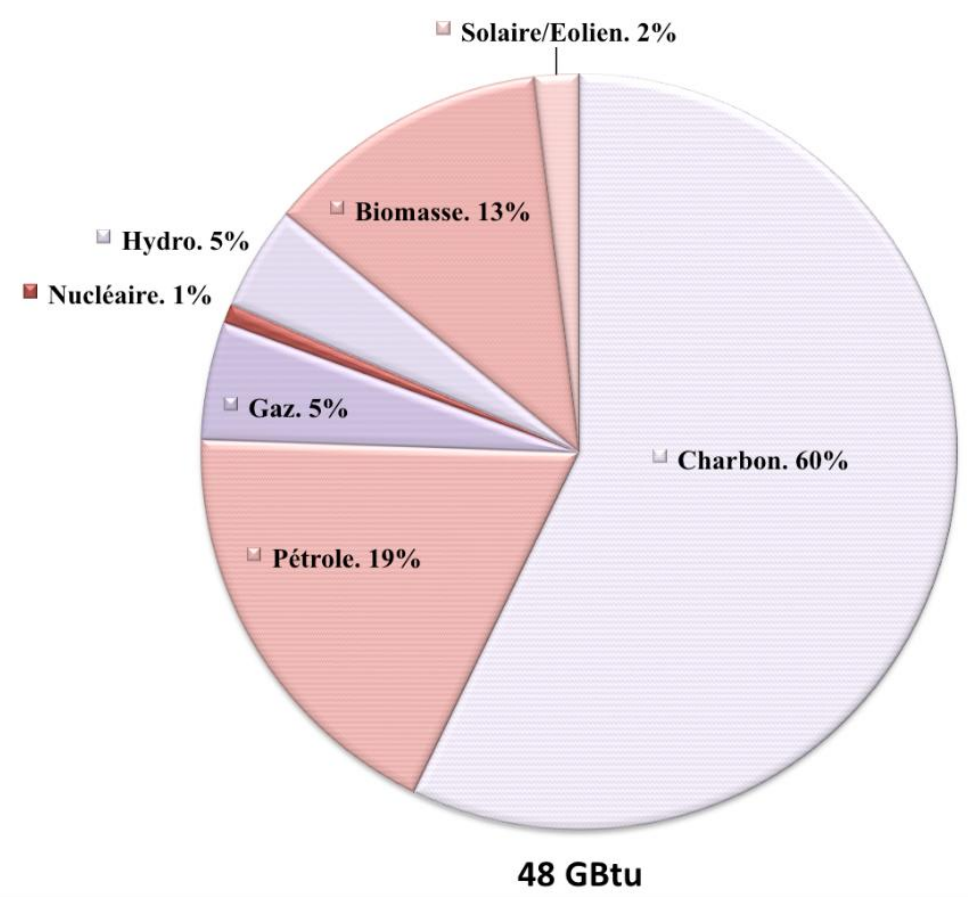

Source: IEA [2016a]. Note: Il s'agit de la part de chaque ressource dans la consommation finale d'énergie.

Une politique de développement des énergies renouvelables agressive. Depuis 2007, une politique d'investissement en énergie renouvelable volontariste est poursuivie. La Chine est le premier investisseur mondial en énergie renouvelable avec 103 milliards de dollars d'investis en 2015 soit une augmentation de 15\% par rapport à l'année précédente (McCrone \& al., 2015). En 2004, la Chine investissait uniquement 2,4 milliards de dollars, soit deux fois moins que les États-Unis et neuf fois moins que l'Europe. Son rattrapage a été spectaculaire étant donné que sur les dix années écoulées, son investissement cumulé a dépassé celui des ÉtatsUnis (388 milliards contre 346 milliards de dollars) (McCrone et al. [2015]). L'énergie hydroélectrique fournit environ $82 \%$ de la consommation électrique provenant d'énergie renouvelable. La capacité installée de production hydroélectrique en Chine était la plus importante au niveau mondial atteignant $250 \mathrm{GW}$ en 2015. A titre de comparaison, la capacité de production de l'énergie hydroélectrique aux États-Unis est de $80 \mathrm{GW}$.

Afin de développer les énergies renouvelables, plusieurs initiatives ont été prises au niveau national. Dans le cadre de la loi d'énergie renouvelable de 2005 suivie par le plan de développement à moyen et long terme des énergies renouvelables, quatre mécanismes ont été mis en place : i) une cible nationale de consommation d'énergie renouvelable révisée à la hausse à chaque plan quinquennal, ii) une connexion minimale et une politique d'achat de l'énergie, iii) des subventions pour l'ensemble les énergies renouvelables (solaire, éolien, biomasse), ${ }^{8}$ iv) un mécanisme de partage des coûts pour le développement des énergies renouvelables (IRENA [2014]). En Mars 2015, le Conseil d'État chinois a annoncé un plan 
pour réformer le secteur électrique en augmentant la part des énergies renouvelables dans la production d'électricité, encourager la concurrence et améliorer l'efficacité. La Chine devrait aussi mettre en place un système national de quotas des émissions de $\mathrm{CO}_{2}$ d'ici 2017. Dans le cadre de cette réglementation, chaque Province devrait fixer un pourcentage minimal de consommation électrique provenant d'énergie renouvelable non hydroélectrique. En fonction du portefeuille énergétique de chaque Province, cette part devrait fluctuer entre $2 \%$ et $10 \%$.

Politique en faveur du gaz de schiste. Le gouvernement envisage de porter la part du gaz naturel dans la consommation énergétique à 10\% en 2020 tout en limitant l'usage du charbon à 5 milliards de tonnes. Au rythme actuel de consommation, les réserves récupérables chinoises en gaz naturel permettraient de satisfaire la demande de gaz au cours uniquement des 24 prochaines années (WEC [2013]). Or, compte tenu de la croissance attendue des besoins énergétiques chinois, les importations de gaz devront augmenter. Le gaz est une ressource plus difficilement transportable que le charbon et, est soumis à de fortes contraintes de capacité. Les importations de gaz peuvent s'effectuer par deux voies. La première, la voie terrestre ou pipeline permet d'importer du gaz de l'Asie Centrale d'une part, et du Myanmar d'autre part (voir Figure 4a). ${ }^{9}$ La capacité maximale d'importation par pipeline est d'environ 31 milliards de mètres cubes en 2015. La seconde, par voie maritime permet de transporter le gaz sous forme liquéfié. Elle offre la possibilité à la Chine de diversifier ses sources d'approvisionnement (Qatar, Indonésie, Malaisie, Australie). La Chine dispose de 10 terminaux méthaniers en opération ayant une capacité maximale de 27 milliards de mètres cube (voir Figure 4a). La Chine est le troisième importateur mondial de gaz liquéfié.

\section{Figure 4 Réserves de gaz de schiste et pipelines chinois}

Figure 4a Pipelines

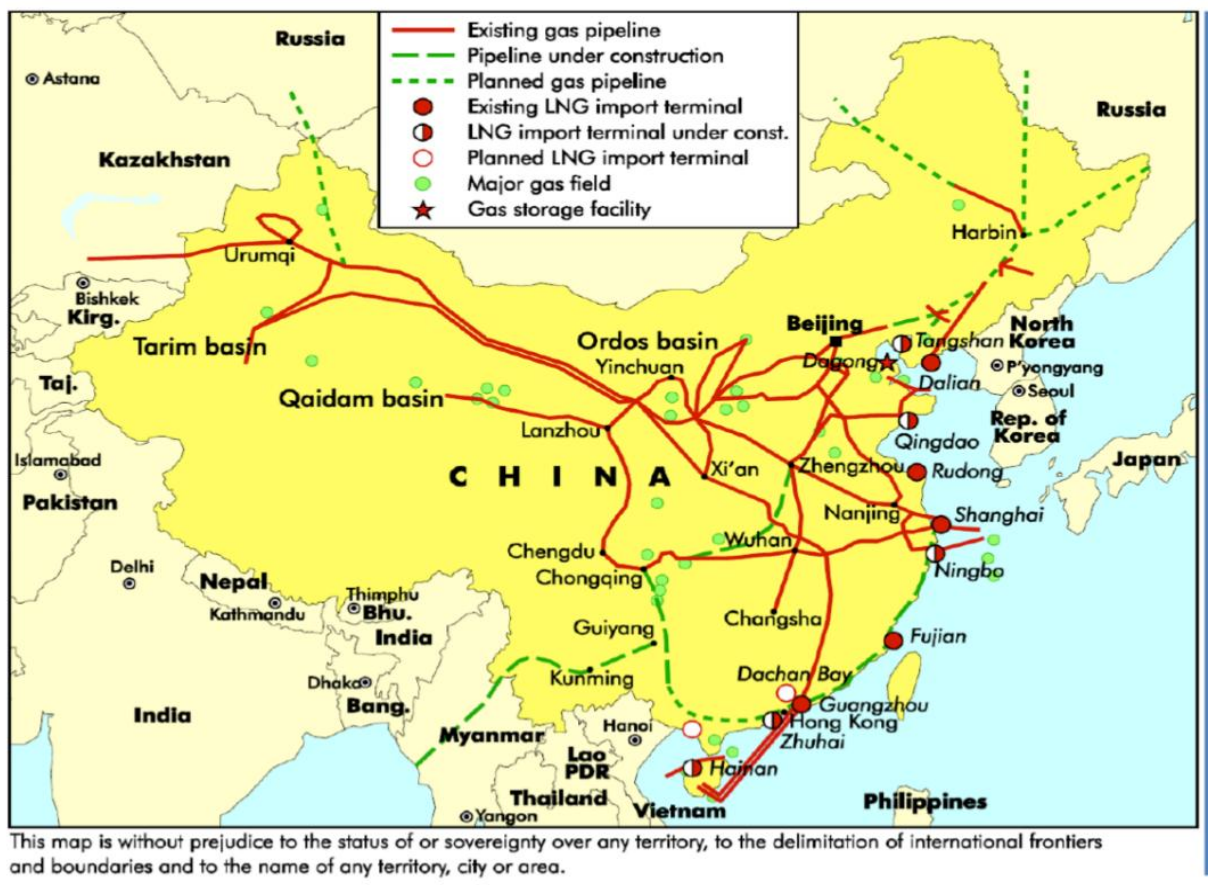


Figure 4b Répartition géographique des réserves

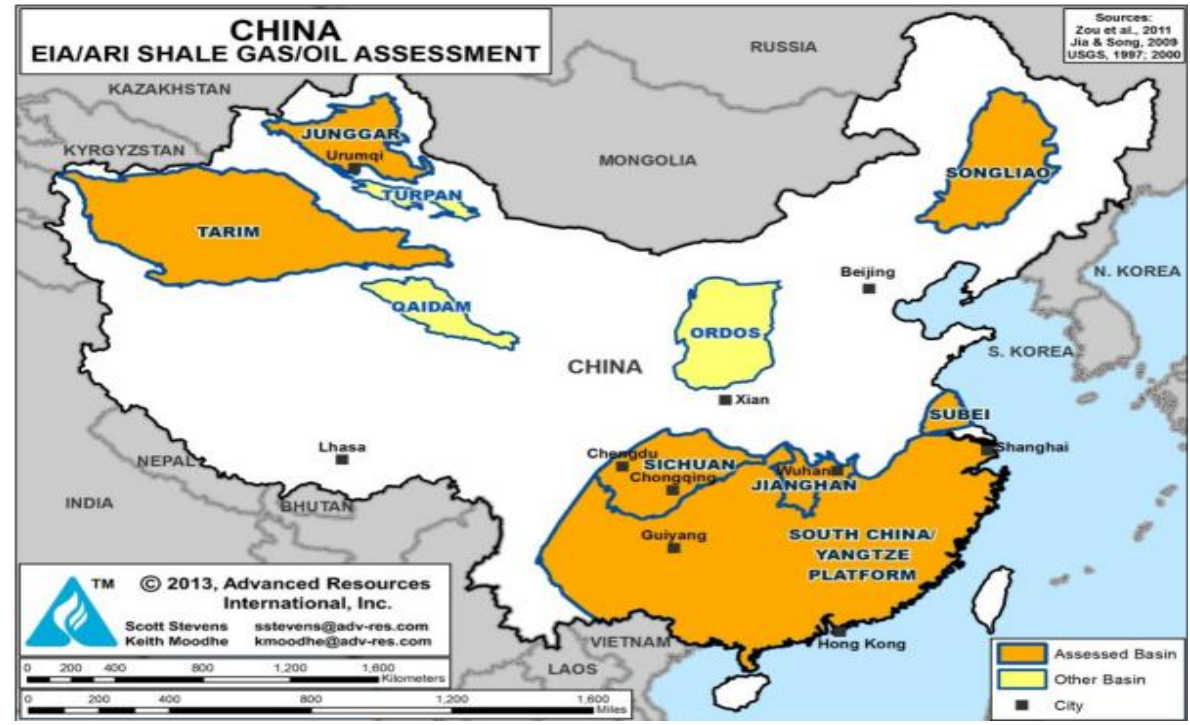

Afin de réduire la part du charbon dans son portefeuille tout en assurant son indépendance énergétique, le gouvernement chinois a décidé dès 2010 de favoriser l'exploitation du gaz de schiste. Les ressources disponibles sont conséquentes, elles représentent environ $20 \%$ des ressources mondiales. Elles permettraient de satisfaire la demande actuelle chinoise en gaz au cours des cent prochaines années. Pour favoriser son exploitation, le gouvernement a réformé le contexte institutionnel et réglementaire $(\mathrm{Hu}$ et $\mathrm{Xu}$ [2013]). En décembre 2011, le gaz de schiste a été reconnu comme une ressource minérale par le Ministère de la Terre et des Ressources chinois ouvrant ainsi la voie à son exploitation et à son développement. L'année suivante, il a autorisé les investissements privés pour son exploitation. Les deux compagnies nationales autorisées à exploiter les réserves : Sinopec et PetroChina ne possédant pas les compétences technologiques, un accord a été signé avec les États-Unis afin de favoriser l'échange de technologies d'exploration et d'exploitation. Le gouvernement dispose d'outils économiques, notamment une subvention de 0,06 dollar par mètre cube de gaz extrait (Xin-Gang et Ya-Hui [2015]). Des diminutions de droit sur les licences d'exploitation sont octroyées. Des exemptions de droits d'importation pour des équipements servant aux industries exploitant le gaz de schiste sont également accordées.

Néanmoins, l'exploitation du gaz de schiste est encore à son balbutiement et rencontre de nombreux obstacles technologiques et géologiques. La fracturation hydraulique est plus difficilement utilisable en Chine (Yuan, et al. [2015]). En effet, les ressources sont situées dans des régions montagneuses et à des profondeurs plus élevées que les ressources nordaméricaines. ${ }^{10}$ En outre, cette technique d'exploitation nécessite de large quantité d'eau or les gisements sont dans des régions arides telles que le Sichuan (voir 
Figure 4b). En outre, des pipelines traversant la Chine doivent transporter le gaz des zones d'extraction du gaz située à l'ouest vers les zones économiques de l'est (voir

Figure 4b). Le développement des gaz de schiste pourrait être limité par le manque de pipelines (Yuan, et al. [2015]).

De fortes incertitudes existent sur le bilan environnemental du gaz de schiste. En comptabilisant uniquement les émissions directes et indirectes de $\mathrm{CO}_{2}$, c'est-à-dire, les émissions au cours de la combustion et celles liées au processus de transformation du gaz en énergie finale, remplacer un MBtu (Million British Thermal Units) d'énergie produit à partir du charbon permet de réduire de $31 \%$ d'émissions de $\mathrm{CO}_{2}$. Toutefois, chaque forage d'énergie fossile présente des risques de fuites de méthane, plus élevés pour le gaz. En comptabilisant les fuites de méthane et les émissions directes et indirectes de $\mathrm{CO}_{2}$, produire un MBtu à partir de gaz de schiste émet deux fois plus de $\mathrm{CO}_{2}$ que de le produire à partir de charbon ( (Allen, et al. [2013]) et (Cathles III, et al. [2012]). En outre, la fracturation hydraulique présente de forts risques pour les ressources en eau, d'une part, du fait des grandes quantités d'eau que cette technologie requiert et d'autre part, des dommages irréversibles qu'elle peut causer aux nappes phréatiques. Au cours du forage, l'eau mélangée à du sable et des additifs chimiques est injectée dans le puits, elle en ressort pour être débarrassée de ses particules et ainsi de suite. Si une fuite a lieu lors de l'injection de l'eau, des polluants peuvent s'infiltrer dans les nappes phréatiques profondes. Pour finir, étant donné que la fracturation hydraulique fracture la roche, elle peut aussi accroître le risque de séismes dans certaines régions sensibles (Jackson, et al. [2014]).

Malgré ces difficultés, la progression de la production de gaz de schiste a été notable. Les compagnies de forage chinoises ont extrait 5 milliards de mètres cubes de gaz de schiste en 2015 représentant $4 \%$ de la production chinoise de gaz. Même si la quantité extraite de gaz de schiste est encore faible comparativement aux besoins énergétiques, elle a enregistré une forte progression au cours de ces dernières années. En effet, elle s'élevait uniquement à 200 millions de mètres cubes en 2013. Les trois-quarts de la production de gaz de schiste sont situés dans la région du Sichuan.

L'énergie nucléaire et la séquestration géologique du carbone. Le gouvernement envisage d'autres options pour atténuer les émissions de $\mathrm{CO}_{2}$ comme le développement de l'énergie nucléaire et la séquestration géologique du carbone, elles font partie des engagements pris dans le cadre de leur contribution nationale ou NDC ( «Nationally Determined Contribution »), c'est-à-dire leur engagement de réduction d'émissions de gaz à effet de serre d'ici 20252030 pris lors des Accords de Paris. Toutefois, aucune cible minimale pour chacune de ces options n'a été spécifiée, seule une feuille de route a été définie.

L'énergie nucléaire est reconnue comme une énergie à faible contenu en carbone. Selon IEA [2015c], son développement a permis d'éviter de relâcher près de $60 \mathrm{Gt}$ de $\mathrm{CO}_{2}$ à travers le monde. ${ }^{11}$ Elle fournissait en 2013 environ $2 \%$ de la consommation primaire d'énergie en Chine. La capacité de production d'énergie à partir du nucléaire était de $17 \mathrm{GW}$ en 2014, 36 réacteurs étaient en opération et 20 en construction (IEA [2015c]). En 2050, la Chine devrait être le leader mondial en termes de capacité de production avec $250 \mathrm{GW}$ installées soit $27 \%$ de la capacité mondiale. IEA [2015c] a estimé que le développement du nucléaire en Chine 
devrait éviter $32 \%$ des émissions de $\mathrm{C}_{2}$ en 2050. L'autre option retenue : la séquestration géologique du carbone devrait permettre de diminuer d'environ $13 \%$ des émissions mondiales en 2050 (IEA [2015b]). ${ }^{12}$

\section{Modèle d'équilibre partiel des marchés énergétiques mondiaux}

Dans cette étude, un modèle d'équilibre partiel du marché mondial de l'énergie à trois régions (Amérique du Nord, Chine et Reste Du Monde -RDM-) est proposé. ${ }^{13}$ La structure du modèle est décrite par la Figure 5.

Description du modèle. L'énergie est produite à partir de ressources fossiles, fissile et renouvelables. Dans le groupe des énergies fossiles sont inclus : le charbon, le pétrole et le gaz naturel conventionnel et non conventionnel (ou gaz de schiste). Parmi les énergies renouvelables, nous distinguons l'énergie hydroélectrique, de la biomasse et des autres énergies renouvelables telles que le solaire et l'éolien. Nous supposons dans cet exercice que la production d'énergie à partir de la biomasse et de l'énergie hydraulique est donnée. En outre, l'électricité peut être produite à partir d'énergie nucléaire.

Le coût d'extraction des ressources fossiles est une fonction croissante et convexe du stock de ressource extrait. ${ }^{14}$ Ainsi, à mesure que les besoins énergétiques s'accroissent, les ressources aux coûts d'extraction plus élevés comme les ressources non conventionnelles deviennent compétitives. Les énergies fossiles peuvent être échangées sur le marché mondial alors que les ressources renouvelables et l'énergie nucléaire sont produites et consommées dans chaque région. Les ressources importées sont des parfaits substituts aux ressources produites dans le pays, en conséquence, les exportations nettes sont définies comme la demande nette de chaque ressource. Les coûts de transport ainsi que les contraintes de capacité pour le transport sont considérés. 
Figure 5 : Description du modèle

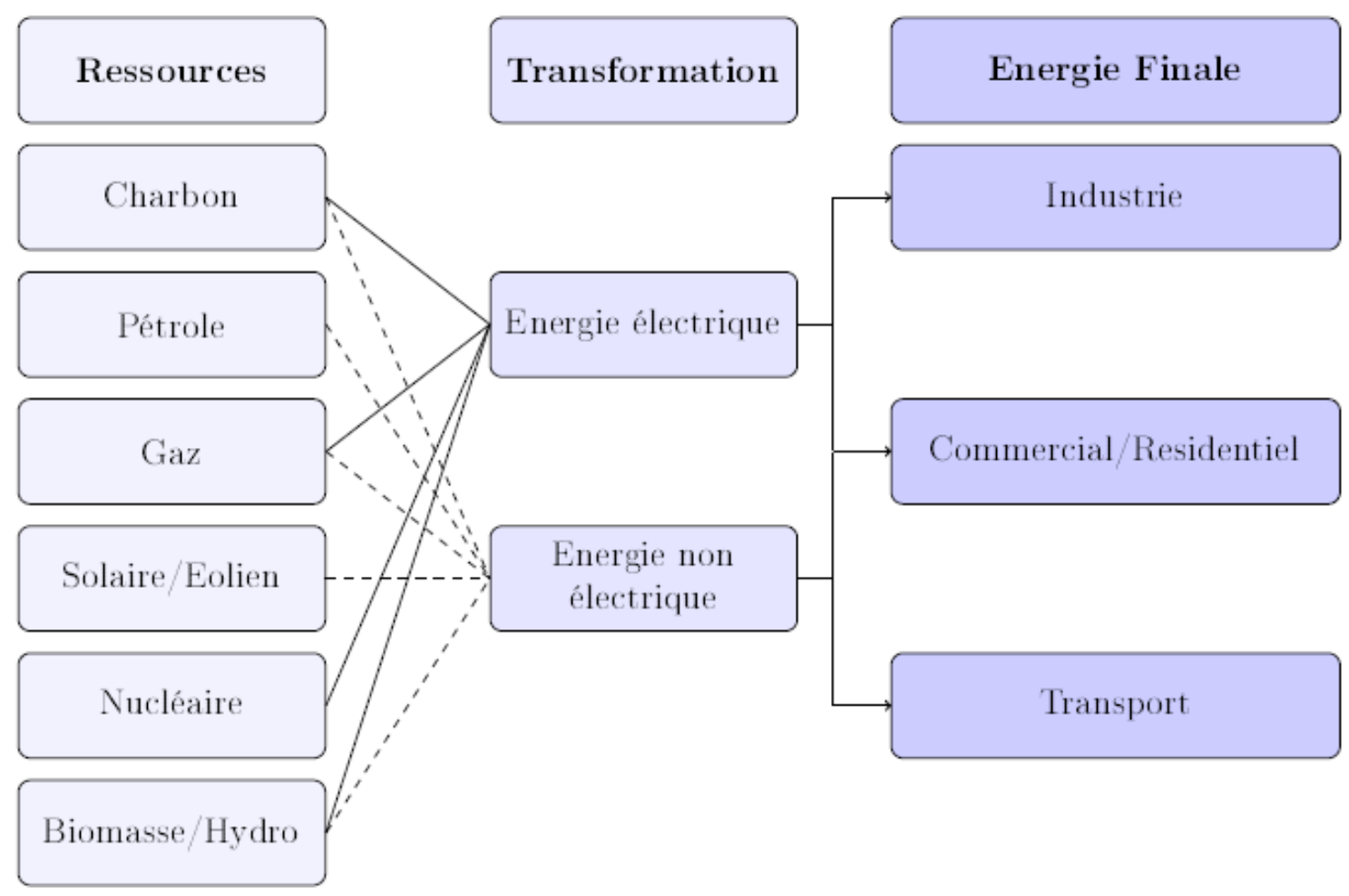

Notes : Le pétrole n'est pas utilisé dans la production d'électricité. Le nucléaire, l'énergie hydroélectrique et l'énergie éolienne et solaire sont uniquement transformés en énergie électrique.

Afin de modéliser correctement le portefeuille énergétique, les différentes ressources sont supposées être imparfaitement substituables. ${ }^{15}$ La production d'énergie se décompose en deux étapes. Dans un premier temps, les ressources peuvent être utilisées soit pour la production électrique soit pour la production directe d'énergie. ${ }^{16}$ Puis, l'électricité et l'énergie produite à partir d'usage directe des ressource participent à la production finale d'énergie permettant ainsi de satisfaire la demande émanant de trois secteurs : industriel, commercial/résidentiel et transport. L'énergie électrique et celle produite à partir d'usage direct des ressources étant des substituts imparfaits, la production finale d'énergie est une fonction de production à élasticité de substitution constante. Pour finir, les émissions de $\mathrm{CO}_{2}$ induites par les différents choix énergétiques sont prises en compte, toutefois, les possibles fuites de méthane ne sont pas considérées (voir Tableau 1). Le taux de progrès technique sur les coûts de production des énergies renouvelables et les coûts de transformation est exogène. ${ }^{17}$

Tableau 1 : Contenu en $\mathrm{CO}_{2}$ des ressources fossiles

\begin{tabular}{cc}
\hline Ressource Fossile & Contenu en $\mathrm{CO}_{2}\left(\mathrm{~kg} \mathrm{CO}_{2} / \mathrm{MBtu}\right)$ \\
\hline Charbon & 95 \\
Gaz Naturel & 54 \\
Gaz de Schiste & 65 \\
Pétrole & 72 \\
\hline
\end{tabular}

Sources : EIA [2013a], Jackson, et al. [2014] et Cathles III, et al. [2012]. 
Le modèle a été calibré pour l'année 2011, il est programmé jusqu'en 2030 en pas de temps d'une année. Le taux d'escompte est de 1.5\% par an (Chakravorty, Magné et Moreaux [2012]). Une présentation détaillée des différentes fonctions utilisées, des méthodes de calibration et des donnés employées est proposée en annexe. ${ }^{18}$

Le planificateur social maximise la somme du surplus des consommateurs et de celui des producteurs sous les contraintes technologiques et celles d'équilibre sur le marché mondial des ressources. Le modèle permet de faire des prévisions sur l'offre et la demande régionales, les échanges mondiaux et le prix de chaque ressource. La production d'électricité et celle d'énergie à partir d'usage de ressources sont endogènes. Enfin, le modèle prévoit la demande finale d'énergie par secteur, d'une part et, le prix de l'énergie finale, d'autre part. La quantité utilisée de chaque ressource dans le portefeuille énergétique dépend du prix relatif des ressources ; elle est d'autant plus élevée que le prix de la ressource est faible comparativement aux autres ressources. La croissance demande d'énergie dépend du taux de croissance du PIB. Le prix de l'énergie finale augmente permettant aux ressources les plus coûteuses telles que les ressources non conventionnelles et les ressources renouvelables de devenir compétitives.

Description des scénarii de politiques énergétique et climatique. Dans le scénario de base, aucune politique énergétique ou climatique n'est introduite dans le modèle. La croissance exogène du PIB accroît la demande d'énergie, quant à l'offre de ressource, elle est modifiée sous l'impulsion du progrès technique. Puis, quatre scénarii sont définis :

1. Le scénario développement du gaz de schiste impose un niveau minimal d'extraction du gaz de schiste en Chine. Nous supposons que la production de gaz de schiste en 2030 doit être multipliée par 10 en 2030 par rapport au scénario de base. A partir de ce scénario, il est possible de déterminer la subvention qui devra être offerte aux propriétaires miniers.

2. Dans le scénario développement des énergies renouvelables, la capacité de production des énergies renouvelables provenant du solaire, de l'éolien doit être au moins égale à $600 \mathrm{GW}$ en 2030.

3. Le scénario stabilisation de la consommation de charbon impose une stabilisation de la consommation chinoise de charbon à partir de 2020 à 4,5 milliards de tonnes.

4. Le scénario taxe sur le $\mathrm{CO}_{2}$ impose une taxe sur chaque tonne d'émission de $\mathrm{CO}_{2}$ de 70 dollars (dollars de 2005 en parité des pouvoirs d'achat).

\section{Résultats}

Pour les différents scénarii, l'analyse se concentre sur l'interprétation des résultats pour l'année 2030 en mettant l'accent sur l'impact des politiques sur les émissions de $\mathrm{CO}_{2}$ chinoises et mondiales. Les fuites de carbone en Amérique du Nord et dans le RDM sont également calculées.

Le scénario développement du gaz. Pour atteindre la cible sur la production minimale de gaz, le gouvernement le subventionne, ainsi, le coût d'extraction du gaz de schiste diminue environ de $40 \%$ par rapport au scénario de base. ${ }^{19}$ Grâce à la subvention offerte aux producteurs, la demande chinoise de gaz augmente, sa part dans la consommation globale d'énergie est portée à $9 \%$ alors qu'elle n'excède pas $3 \%$ dans le scénario de base. ${ }^{20}$ Le prix du 
gaz diminue entrainant une baisse du prix de l'énergie finale dans les secteurs commercial et résidentiel et dans le secteur de l'industrie diminuent respectivement de 3\% et 5\%. La baisse de prix est plus marquée pour l'électricité, le prix diminue de $15 \%$. La demande d'énergie finale augmente comparativement au scénario de base (voir Figure 6). Même si la part de la consommation de gaz de schiste augmente au détriment de celle du charbon, les émissions de $\mathrm{CO}_{2}$ chinoises croissent de $5 \%$ (voir Figure 7). ${ }^{21}$ L'effet demande : augmentation de la demande finale d'énergie l'emporte sur l'effet offre : substitution d'une énergie moins polluante à une énergie plus polluante.

En outre, les émissions de $\mathrm{CO}_{2}$ dans les autres régions augmentent. En effet, suite à la baisse de la demande chinoise de charbon, le prix mondial du charbon diminue de $5 \%$ et sa consommation progresse dans le RDM. Par conséquent, les émissions augmentent de 3\% en Amérique du Nord et de 1\% dans le RDM (voir Figure 8). L'augmentation des émissions en Amérique du Nord (respectivement RDM) représente 60\% (respectivement 19\%) de l'augmentation des émissions en Chine (voir Figure 9).

Figure 6 : Demande finale d'énergie en Chine sous différents scénarii en 2030

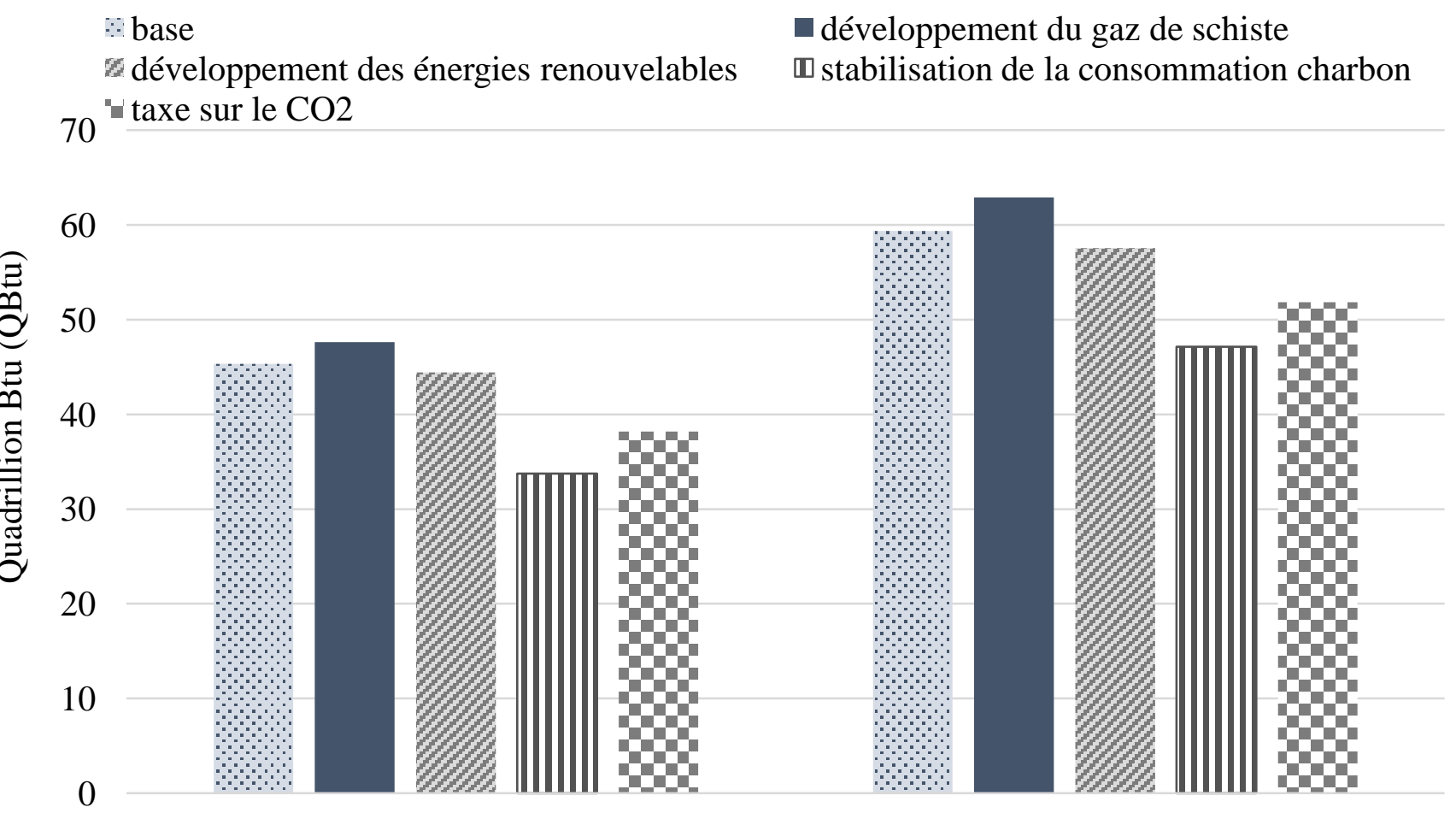

Industrie

Commercial/Residentiel 
Notes : Les résultats pour le secteur des transports ne sont pas reportés étant donné que ce dernier est moins affecté par les politiques mises en places. De plus, la part du gaz dans ce secteur étant minime, les résultats ont moins d'intérêt dans le cadre de cette étude.

Le scénario développement des énergies renouvelables. Dans ce scénario, une cible de consommation minimale de l'énergie renouvelable à partir de l'éolien et du solaire est définie. En incitant les consommateurs à adopter une énergie plus onéreuse, le gouvernement participe à l'accroissement du prix de l'énergie finale conduisant ainsi à une diminution de la consommation d'énergie finale (voir Figure 6). La baisse de la demande d'énergie finale combinée à une recomposition du portefeuille énergétique en faveur d'énergies vertes. Ainsi, les émissions de $\mathrm{CO}_{2}$ baissent de $8 \%$.

Les émissions de $\mathrm{CO}_{2}$ dans les autres régions. Le prix mondial de toutes les énergies fossiles diminue avec une baisse plus accentuée pour le charbon. Leur part dans le portefeuille énergétique augmente entrainant une hausse de $1 \%$ des émissions par rapport au scénario de base (voir Figure 8). L'augmentation des émissions de $\mathrm{C}_{2}$ en Amérique du Nord et dans le RDM représente environ $12 \%$ de la réduction des émissions chinoises (voir Figure 9).

Le scénario stabilisation de la consommation de charbon. Dans ce scénario, le gouvernement chinois impose une stabilisation de la consommation de charbon à 4,5 milliards de tonnes. En imposant une telle contrainte, tout se passe comme si le gouvernement chinois taxait le charbon. Le prix domestique du charbon augmente de $30 \%$ par rapport au scénario de

Figure 7 : Variation des émissions de $\mathrm{CO}_{2}$ chinoises en \% sous les différents scénarii comparativement au scénario de base en 2030

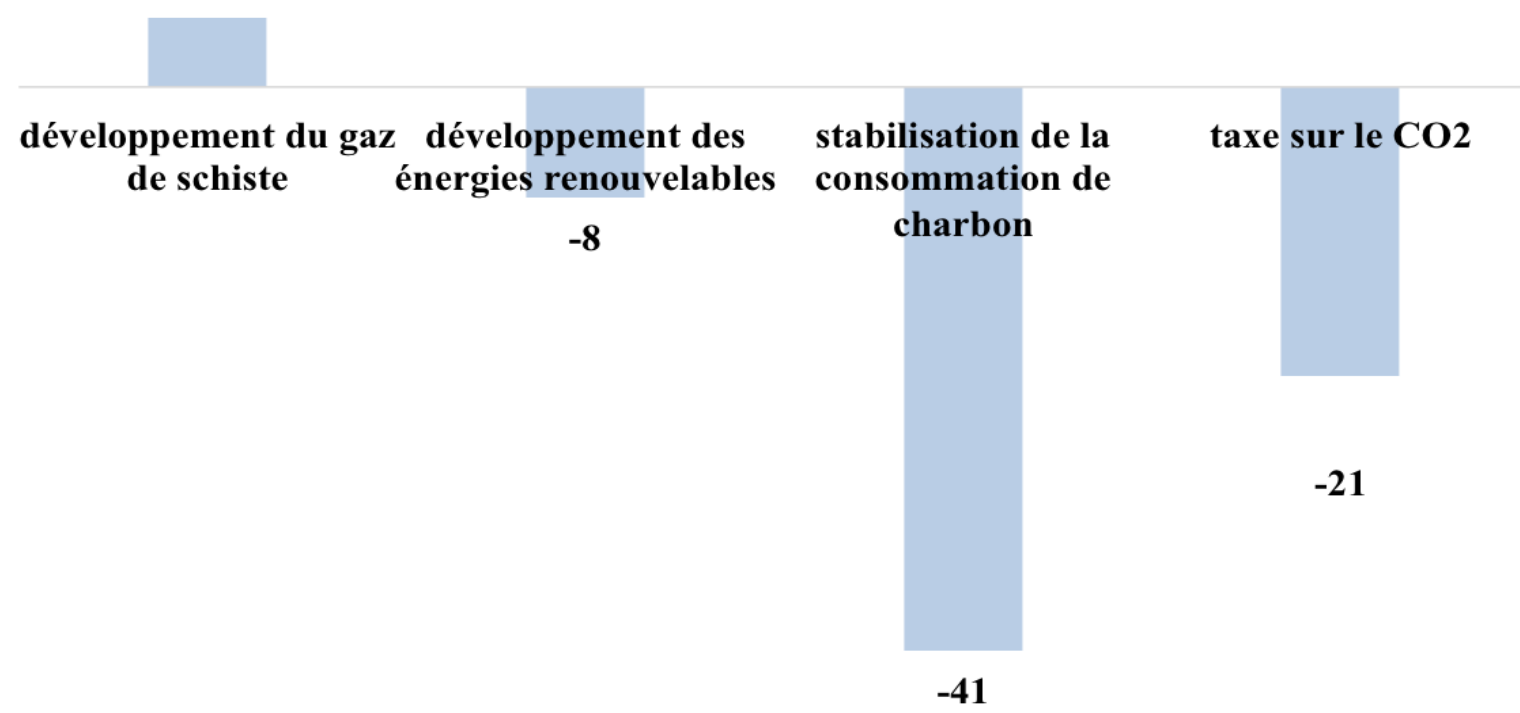


base et sa consommation diminue d'un peu plus d'un tiers. Pour comprendre l'effet net de cette politique sur les émissions de $\mathrm{CO}_{2}$, nous devons en comprendre l'effet sur le portefeuille énergétique, d'une part, et sur la demande finale d'énergie, d'autre part. Le charbon étant la ressource la plus polluante, toute politique taxant son usage favorise le développement d'énergie moins polluante. Dans ce scénario, le principal bénéficiaire est le gaz de schiste. La consommation d'énergies renouvelables telles que l'éolien/solaire n'est pas affectée. La taxe implicite sur le charbon accroît le prix de l'énergie finale ainsi que celui de l'électricité. La demande finale d'énergie décroît (voir Figure 6). Les émissions de $\mathrm{CO}_{2}$ chinoises diminuent de $41 \%$ par rapport au modèle de base (voir Figure 7).

En revanche, les émissions de $\mathrm{CO}_{2}$ en Amérique du Nord (respectivement RDM) augmentent de $2.6 \%$ (respectivement de 3.3\%) (voir Figure 8). Les fuites de $\mathrm{CO}_{2}$ sont positives. L'accroissement des émissions de $\mathrm{CO}_{2}$ en Amérique du Nord (respectivement RDM) représente $6 \%$ (respectivement $8 \%$ ) de la diminution des émissions de $\mathrm{CO}_{2}$ en Chine (voir Figure 9).

Le scénario taxe sur le $\mathrm{CO}_{2}$. Le gouvernement chinois impose une taxe de 70 dollars sur chaque émission de $\mathrm{CO}_{2}$ en 2030. De ce fait, ces dernières diminuent de $20 \%$ par rapport au scénario de base. Chaque ressource énergétique étant taxée à hauteur de son contenu en $\mathrm{CO}_{2}$, le prix du charbon augmente plus que celui des autres ressources. La consommation de toutes les énergies fossiles diminue, seule celle des énergies renouvelables (éolien/solaire) croît.

Figure 8 : Variation des émissions de C02 (\%) en Amérique du Nord et dans le RDM

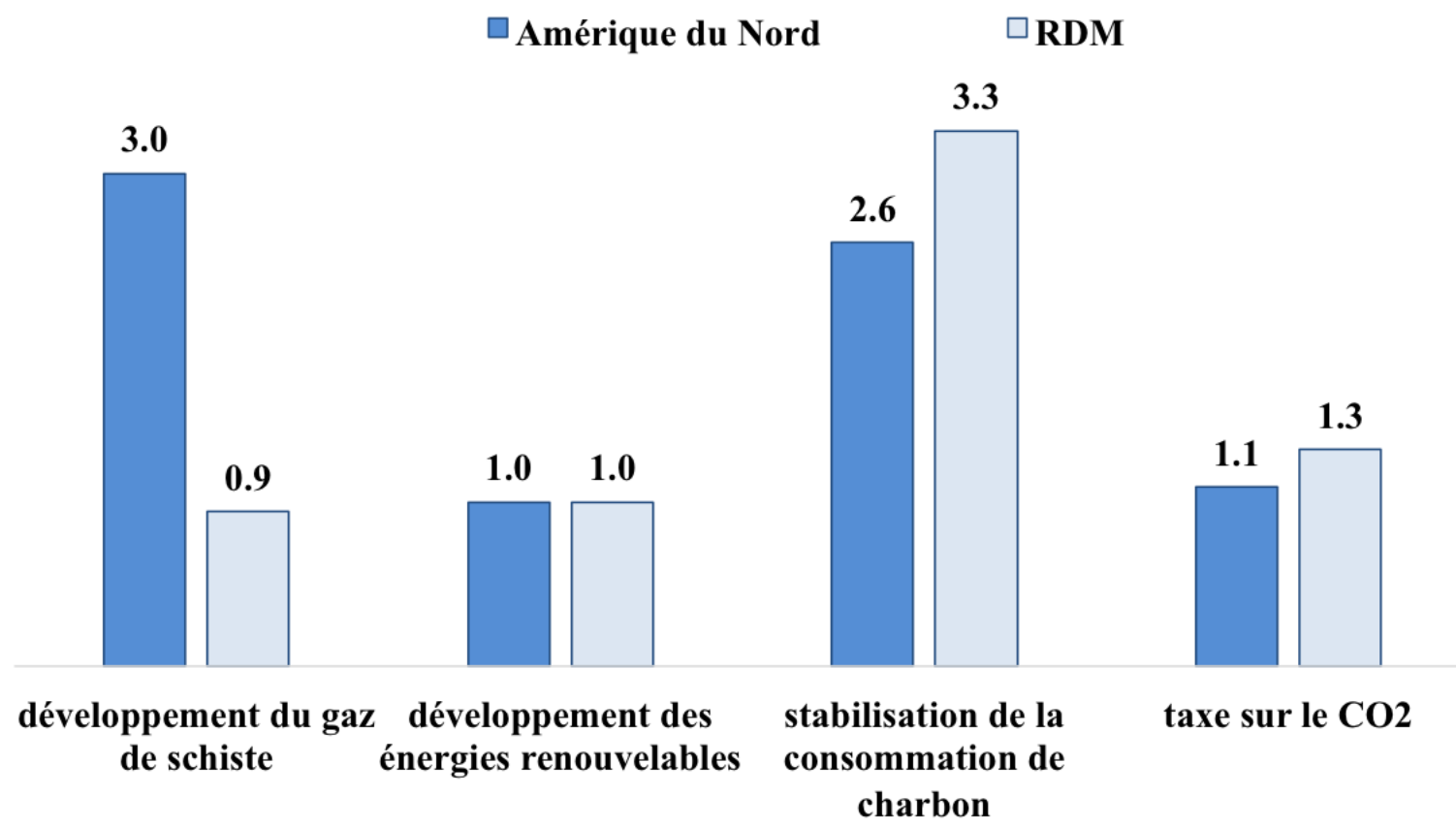


Contrairement à d'autres études, Henriet et Schubert [2015] et Daubanes, Henriet et Schubert [2016], notre étude montre que la politique climatique ne favorise pas le développement du gaz de schiste. La taxe implicite sur le C02 freine la demande d'énergie finale (voir Figure 6). Le prix de l'énergie finale augmente d'environ $30 \%$ pour tous les secteurs. Les émissions mondiales de $\mathrm{CO}_{2}$ croissent (voir Figure 8).

\section{Figure 9 : Fuites de $\mathrm{C02}$ en \% en Amérique du Nord et dans le RDM}

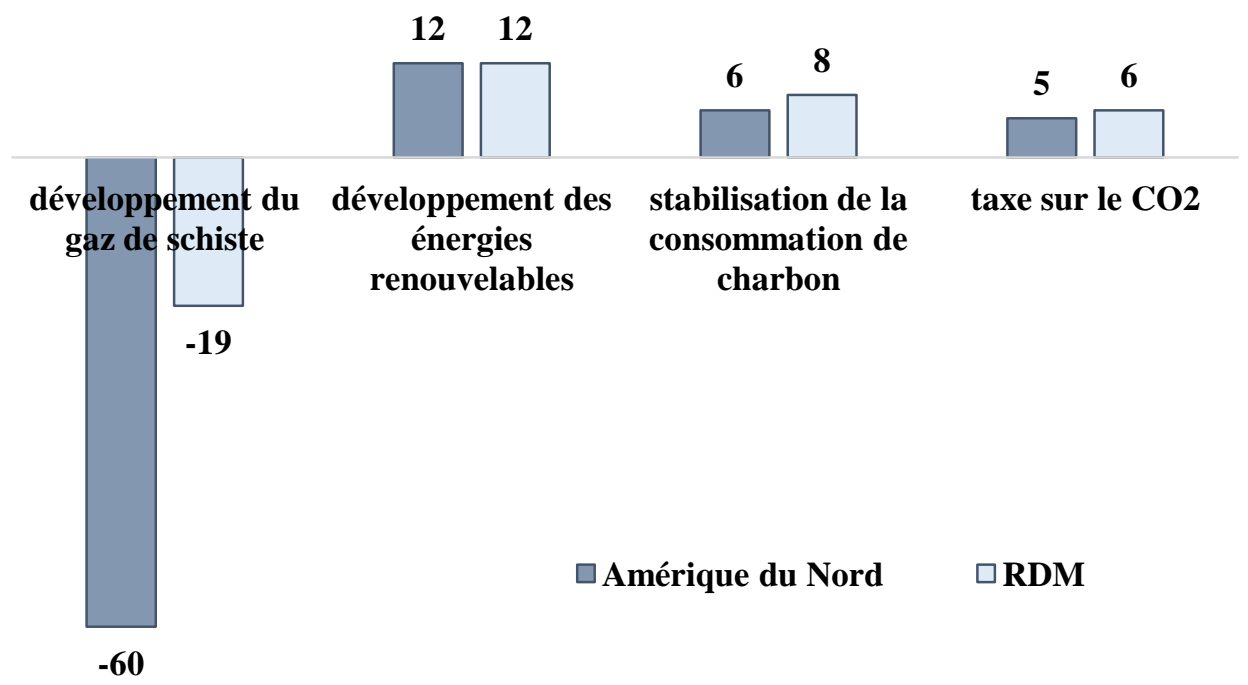

Notes : Les fuites sur le C02 sont calculées comme le rapport entre la variation en pourcentage des émissions de C02 en Amérique du Nord et dans le RDM par rapport au scénario de base et la baisse en pourcentage des émissions de CO2 en Chine par rapport au scénario de base, multiplié par 100.

\section{Conclusion}

L'économie chinoise est gourmande en charbon, en effet, environ $60 \%$ de son énergie provient de cette ressource. Afin de limiter voire de stabiliser ses émissions futures de $\mathrm{CO}_{2}$, le gouvernement chinois cherche des substituts moins polluants. Une ressource permettrait non seulement de préserver l'indépendance énergétique chinoise tout en atténuant ses émissions : le gaz de schiste. Dans cette étude, nous avons développé un modèle d'équilibre partiel des marchés énergétiques mondiaux afin d'appréhender l'opportunité pour le gouvernement chinois de développer cette énergie. Nos résultats montrent que le développement à grande échelle du gaz de schiste ne pourra se réaliser qu'à l'aide de fortes subventions à la production. En effet, la part du gaz de schiste dans la consommation d'énergie en Chine pourrait atteindre presque $10 \%$ si son coût diminuait d'un tiers. Cette politique, en offrant une subvention à la production, participerait à la diminution du prix de l'énergie et contribuerait donc à accroître les émissions chinoises de $\mathrm{CO}_{2}$. Une politique favorisant le développement des énergies renouvelables permettrait de réduire les émissions chinoises de $\mathrm{CO}_{2}$ de $8 \%$. Une taxe sur le $\mathrm{CO}_{2}$ ne favoriserait pas le développement du gaz de schiste mais bénéficierait aux énergies renouvelables. Ces différentes politiques induisent une augmentation notable des émissions de $\mathrm{CO}_{2}$ en Amérique du Nord et dans le RDM. 
Cette recherche pourrait bénéficier de nombreux prolongements. Premièrement, les résultats de cette étude sont fortement corrélés aux élasticités de demande et d'offre (Daubanes, Henriet et Schubert [2016]). Selon l'échantillon choisi et la méthode d'estimation utilisée, les élasticités peuvent être différentes et impacter fortement les résultats des modèles de simulation. Afin de remédier à ce biais, des méthodes de Monte-Carlo peuvent être utilisées (Chakravorty, Hubert et Ural-Marchand [2016]). Deuxièmement, les dommages environnementaux du gaz de schiste ainsi que les barrières technologiques au développement du gaz de schiste ont été ignorées. Troisièmement, un progrès technique exogène est une hypothèse fortement réductrice sachant que ce dernier peut non seulement améliorer les coûts de production des énergies vertes mais aussi diminuer les coûts d'extraction des ressources non conventionnelles (Chakravorty, Fischer et Hubert [2016]).

\section{Références}

Allen, D. T., Torres, V. M., Thomas, J., Sullivan, D. W., Harrison, M., Hendler, A., . . al., e. (2013). Measurements of methane emissions at natural gas production sites in the United States. Proceedings of the National Academy of Sciences, 110(44), 1777817773.

Auffhammer, M., \& Gong, Y. (2015). China's Carbon Emissions from Fossil Fuels and Market-Based Opportunities for Control. Annual Review of Resource Economics, 7(1), 11-34.

Berntein, M., \& James, G. (2005). Regional Differences in the Price-Elasticity of Demand for Energy. National Renewable Energy Laboratory .

Cathles III, L. M., Brown, L., Taam, M., \& Hunter, A. (2012). A commentary on "The greenhouse-gas footprint of natural gas in shale gas formations" by R.W. Howart, R. Santoro, and Anthony Ingraffea. Climatic Change, 113(2), 525-535.

Chakravorty, U., Fischer, C., \& Hubert, M.-H. (2016). Post-Paris Clean Energy Options for China. Mimeo.

Chakravorty, U., Hubert, M.-H., \& Ural-Marchand, B. (2016). The effect of US biofuel mandate on poverty in India. Working Paper.

Chakravorty, U., Magné, B., \& Moreaux, M. (2012). Resource Use under Climate Stabilisation: Can Nuclear power Provide Clean Energy? Journal of Public Economics, 14(2), 349-389.

Chen, X., Huang, H., Khanna, M., \& Önal, H. (2014). Alternative transportation fuel standards: Welfare effects and climate benefits. Journal of Environmental Economics and Management, 67(3), 241-257.

Cullen, J., \& Mansur, E. (2016). Will Carbon Prices Reduce Emissions in the U.S. Electricity Indusctry? Evidence from the Shale Gas Experience. Wroking Paper. 
Daubanes, J., Henriet, F., \& Schubert, K. (2016). More gas to reduce CO2 emissions. Working Paper .

EIA. (2013a). Carbon Dioxyde Emissions Coefficients. Energy information Administration .

EIA. (2013b). Technically Recoverable Shale Oil and Shale Gas Resources: An Assessment of 137 Shale Formations in 41 Countries Outside the U.S. Washington: Energy Information Administration .

EIA. (2014a). International Energy Outlook. Washington, DC: Energy Information Administration .

EIA. (2014b). Model Documentation Report: Industrial Demand Module of the National Energy Modeling System. Washington, DC: Energy Information Administration.

EIA. (2015a). Four Countries Added to Global Shale Oil and Natural Gas Resource Assessment. Washington, DC: Energy Information Administration.

EIA. (2015b). Shale Gas Production. Washington: Energy Information Administration.

EIA. (2016). International Energy Statistics. Washington, DC: Energy Information Administration.

Fischer, C., \& Salant, S. W. (2012). Alternative Climate Policies and Intertemporal Emissions leakage: quantifying the Green Paradox. Resources for the Future Discussion paper(12-16).

Hamilton, J. D. (2009). Understanding Crude Oil Prices. The Energy Journal, 30(2), 179206.

Henriet, F., \& Schubert, K. (2015). Should We Extract the European Shale Gas? The Effect of Climate and Financial Constraints . Working Paper.

Howarth, R. W., Ingraffea, A., \& Engelder, T. (2011). Natural gas: Should fracking stop? Nature, 477(7364), 271-275.

Hu, D., \& Xu, S. (2013). Opportunity, challenges and policy choices for China on the devlopment of shale gas. Energy Policy, 60, 21-26.

IEA. (2010). Projected Costs of Generating Electricity, 2010 Edition. Paris : International Energy Agency.

IEA. (2012). Technology Roadmap China Wind Energy Development Roadmap 2050. OECD/International Energy Agency and Energy Research Institute .

IEA. (2013). Resources to Reserves, 2013. Paris: Inetrnational Energy Agency.

IEA. (2013b). Energy Balances of OECD Countries. Paris : International Energy Agency.

IEA. (2014). Prices and taxes statistics. Paris: International Energy Agency .

IEA. (2015a). CO2 Emissions from Fuel Combustion: Highlights. Paris : International Energy Agency . 
IEA. (2015b). Energy Technology Perspectives 2015 - Mobilising Innovation to Accelerate Climate Action. paris : International Energy Agency .

IEA. (2015c). Technology Roadmap: Nuclear Energy. Paris : International Energy Agency.

IEA. (2016a). Energy Blances of Non OECD Countries. Paris: International Energy Agency.

IRENA. (2014). Renewable Energy Prospects: China. Abu Dhabi: International Renewable Energy Agency .

Jackson, R. B., Vengosh, A., Carey, J., Davies, R., Darrah, T., O'Sullivan, F., \& Pétron, G. (2014). The Environmental Costs and Benefits of Fracking. Annual Review of Environment and Resources, 39, 327-362.

LBNL. (2014). Key China Energy Statistics. Lawrence Berkeley National Laboratory .

Lin, C., \& Zeng, J. (2013). The Elasticity of Demand for Gasoline in China . Department of Agriculture and Resource Economics.

McCrone, A., \& al., e. (2015). Global Trends in Renewable Energy Investment 2015. Frankfurt School of Finance \& Management and United Nations Environment Programme .

Phounin, H., \& Shigeru, K. (2014). Analysis on Price Elasticity of Energy Demand in East Asia: Empirical Evidence and Policy Implications for ASEAN and East Asia. ERIA Discussion Paper Series. April.

Rutherford, T. (2002). Lecture notes on constant elasticity functions. Working Paper, University of Colorado.

Wang, Z., \& Krupnick, A. (2013). A Retrospective Review of Shale Gas Development in the United States: What Led to the Boom? Resources for the Future Discussion Paper 1312 .

WEC. (2013). World Energy Resources: Survey 2013 . London: World Energy Council.

World Bank. (2016). World Databank. Washington : World Development Indicators, World Bank.

Xin-Gang, Z., \& Ya-Hui, Y. (2015). The current situation of shale gas in Sichuan, China. Renewable and Sustainable Energy Reviews, 50, 653-664.

Yuan, J., Luo, D., Xia, L., \& Feng, L. (2015). Policy recommendations to promote shale gas development in China based on technical and economic evaluation. Energy Policy, 85, 194-206.

\footnotetext{
${ }^{2}$ La marne est une roche sédimentaire, mélange de calcaire et d'argile dans des proportions à peu près équivalentes allant de $35 \%$ à $65 \%$.

${ }^{3}$ Le principe est simple : après un forage vertical pour atteindre la couche de schiste, on pénètre la roche horizontalement, puis on injecte à haute pression (600 bars) des milliers de
} 
litres d'eau (de 7000 à 15 000) chargée en sable et en adjuvants chimiques pour fracturer la roche et pousser le gaz vers la surface. Lors de l'extraction, les trois-quarts du liquide de fracturation (mélange d'eau, de sable et de produits chimiques) restent dans la couche de schiste, le quart récupéré en surface doit être évacué vers des usines de retraitement.

${ }^{4} 95$ tonnes de $\mathrm{CO}_{2}$ sont émises pour produire un million de British Thermal Units (MBtu) à partir de charbon alors que 65 tonnes sont émises si le gaz de schiste est utilisé (voir Tableau $1)$.

${ }^{5}$ La fuite inter-temporelle du $\mathrm{CO}_{2}$ peut être observée si un pays favorise via des subventions des énergies fossiles à plus faible contenu en $\mathrm{CO}_{2}$. Par exemple, les émissions de gaz de schiste pour produire une unité d'énergie sont inférieures d'un tiers à celle du charbon. Si le gaz de schiste est subventionné, son prix diminue et sa demande augmente. Les ressources renouvelables deviennent relativement moins compétitives, ce qui retarde leur pénétration du marché. La fuite spatiale du $\mathrm{CO}_{2}$ est observée si à la suite d'une politique climatique dans un pays ayant un pouvoir de marché sur les marchés énergétiques mondiaux, les émissions de $\mathrm{CO}_{2}$ augmentent dans le reste du monde. Le pays régulateur doit avoir un pouvoir de marché sur les marchés énergétiques mondiaux. Illustrons nos propos en prenant comme exemple la Chine qui consomme 50\% du charbon mondial (IEA [2016b]). Si la Chine taxe les émissions de $\mathrm{CO}_{2}$, sa consommation de charbon devrait diminuer entrainant une diminution de la demande mondiale. Le prix mondial du charbon décline, les autres pays sont incités à accroître leur consommation de charbon si aucune politique taxant les émissions de $\mathrm{CO}_{2}$ est en place. Leurs émissions augmentent.

${ }^{6}$ L'intensité en $\mathrm{CO}_{2}$ du PIB est calculée en utilisant le PIB en parité de pouvoir d'achat en dollar de 2005. Toutes les données sur les émissions de $\mathrm{C}_{2}$ sont extraites de (IEA, 2015a). ${ }^{7}$ En 2013, l'intensité en $\mathrm{C}_{2}$ de la Chine avait diminué de $20 \%$ par rapport à son niveau de 2005 (IEA 2015a).

${ }^{8}$ Par exemple, la subvention accordée à l'énergie solaire varie de 0.145 US $\$ / \mathrm{kWh}$ à 0.161 US $\$ / \mathrm{kWh}$ en fonction de la province (IRENA, 2014).

${ }^{9}$ Gazprom et la société chinoise China National Petroleum Corporation (CNPC) ont signé un accord en 2014 pour la construction d'un pipeline qui permettra d'acheminer environ 38 milliards de mètres cube de gaz de la Sibérie vers la Chine d'ici 2017.

${ }^{10}$ Certaines ressources chinoises sont situées à plus de 2500 mètres de profondeur rendant la fracturation hydraulique plus complexe. De ce fait, le coût d'extraction du gaz de schiste est plus élevé.

${ }^{11}$ Toutefois, l'énergie nucléaire présente de nombreux risques : recyclage des déchets, dommages irréversibles causés à l'environnement en cas d'accident nucléaire.

${ }^{12}$ Dix pays dont la Chine, le Canada, la Norvège ont explicitement inclus les technologies de capture et de stockage du carbone dans la définition de leur INDC.

${ }^{13}$ La région de l'Amérique du Nord comprend : les États-Unis, le Canada et le Mexique.

${ }^{14} \mathrm{La}$ fonction de coût d'extraction est décrite par l'équation A 1 de l'Appendice (Chakravorty, Magné et Moreaux [2012]).

${ }^{15}$ La production d'énergie est modélisée via une fonction de production à élasticité de substitution constante (voir Appendice pour le détail de la calibration de la fonction de production ainsi que la valeur de l'élasticité de substitution).

${ }^{16}$ Le raffinage du pétrole pour produire de l'essence ou du diesel est un exemple d'usage direct des ressources.

${ }^{17}$ Le progrès technique affecte le coût de production des énergies renouvelables ainsi que les coûts de transformation des ressources en énergie finale. Dans ce modèle simplifié, les 
améliorations techniques permettant de diminuer le coût d'extraction des ressources et d'améliorer le rendement des gisements est ignoré.

${ }^{18}$ Pour une version très détaillée de l'ensemble des données utilisées et un descriptif minutieux des méthodes de calibration, le lecteur est invité à lire (Chakravorty, Fischer et Hubert [2016]).

${ }^{19}$ En 2030, le coût d'extraction du gaz de schiste devrait atteindre hors subvention 6 US dollars par MBtu.

${ }^{20}$ Dans le scénario de base, le développement du gaz en Chine est paralysé par la faiblesse des coûts de production du charbon.

${ }^{21}$ Produire une unité d'énergie à partir de gaz de schiste en remplacement d'une unité d'énergie produite à partir de charbon permet de diminuer les émissions de $\mathrm{C}_{2}$ d'environ un tiers (voir Tableau 1). 